\title{
Continuation of periodic solutions for systems with fractional derivatives
}

\author{
Pierre Vigué, Christophe Vergez, Bruno Lombard, Bruno Cochelin \\ Aix Marseille Univ, CNRS, Centrale Marseille, LMA, Marseille, France \\ \{vigue, vergez, lombard\} @lma.cnrs-mrs.fr, bruno.cochelin@centrale-marseille.fr
}

\begin{abstract}
This paper addresses the numerical computation of periodic solutions of nonlinear differential systems involving fractional derivatives. For this purpose, the Harmonic Balance Method and the Asymptotic Numerical Method are combined, generalizing an approach largely followed in non-fractional systems. This enables to perform the continuation of periodic solutions of fractional systems with respect to a system parameter or to the fractional order. In the particular case of a constant fractional order, the results are validated by a successful comparison with an alternative formulation based on the diffusive representation of fractional operators. The new numerical strategy presented here allows to simulate phenomena still lacking from theoretical foundations. For example, the numerical experiments proposed here lead to a bifurcation similar to the Hopf bifurcation, well known in the case of non-fractional systems. Throughout this article, the Weyl derivative is used; its link with the classic Caputo derivative is elucidated.
\end{abstract}

\section{Introduction}

Time-periodic solutions are commonly investigated in dynamical systems. They can be an issue, causing noise or destructive motions (for example in rotating machinery [1]) or they can be sought after (for example, auto-oscillations of winds and bowed strings instruments [2]). The continuation of periodic solutions produces bifurcation diagrams representing the existence, stability and other characteristics of such periodic solutions (amplitude, angular frequency, etc) with respect to some parameters of the nonlinear system. For dynamical systems represented by standard differential equations (i.e. without fractional derivatives), many numerical methods have been developed to compute bifurcation diagrams through numerical continuation [3]. The association of the Asympotic Numerical Method (ANM), a continuation technique based on Taylor series, with the Harmonic Balance Method (HBM), proved its effectiveness to continue periodic solutions of standard nonlinear differential systems, either forced or autonomous [4].

However, many dynamical systems issued from physics are non-standard in the sense that they also include fractional derivatives. It is notably the case of bowed string instruments, where models have been proposed to take into account the rosin in the contact zone between the bow hair and the string [5]. Heat exchanges at the bow/string contact point can be reformulated as a half-derivative in time ([6], section 8.4). Propagation of finite-amplitude acoustic waves in guides provides a second example of systems containing nonlinearity and fractional derivative; it occurs in wind instruments of the brass family $[7,8]$ and in the modeling of acoustic solitons [9]. More generally, fractional operators are also used in a variety of models in physics (viscoelasticity [10], Fokker-Planck equation [11], advection-dispersion equation [12], solid mechanics [13], etc) and other fields $[14]$.

Time-periodic solutions of dynamical systems with fractional derivatives constitute an open subject of research, both from a mathematical and numerical point of view. The mathematical issues, concerning e.g. the definitions of periodicity, are out of the scope of this work; the interested reader is refered to [15-20] for an introduction to these topics. In-depth theory of fractional calculus and dedicated numerical analysis are widely studied in many papers, such as [21-29].

In the present paper, we focus on the effective computation of periodic solutions and on their continuation. The principle of coupling ANM and HBM is thus extended and applied to non-standard dynamical systems with fractional operators. To our knowledge, this topic has not been addressed in the literature, despite its relevance. 
The paper is organized as follows. In section 2, the ANM-HBM framework is recalled in the standard framework. Two definitions of the fractional derivative are recalled: the Caputo derivative [30], usual but unsuited to the calculation of periodic solutions; the Weyl derivative [26], less usual but directly applicable to the case treated here. The section 2 concludes by emphazing the link between these two definitions, which constitutes a first original contribution of the article.

The core of the paper is made up of the sections 3 and 4, which detail two methods for the continuation of periodic solutions of fractional systems. In section 3, the ANM-HBM framework introduced in [4] is extended to a new method called Harmonic Balance with Weyl derivative (HBW). This method is able to continue periodic solutions of nonlinear differential systems containing Weyl fractional terms. The HBW approach allows the continuation of the solutions with respect to all the parameters of the problem, including the order of fractional derivative. However, it does not give access to stability results. Section 4 is dedicated to an alternative approach, based on the so-called diffusive approximation. This second approach amounts to writing an approximate system with usual (non-fractional) derivatives, for which stability results are naturally obtained. However, it cannot be applied when the continuation parameter is the order of fractional operator.

In section 5, the two aforementioned methods are applied to the generic case of the fractional Van der Pol oscillator. When the fractional order is constant, they are successfully compared. Since using the HBW does not require to approximate the fractional derivatives, it can provide more accurate results on the system than the diffusive approximation (for example, the location of a Hopf bifurcation). The section 6 concludes the paper, and technical mathematical details are given in appendices.

\section{General framework}

\subsection{Continuation of periodic solutions: coupling the ANM and the HBM}

The continuation of periodic solutions of nonlinear systems

$$
\dot{\mathbf{x}}=\mathbf{f}(\mathbf{x}, \lambda)
$$

can be performed by coupling two methods: the Harmonic Balance Method (HBM) and the Asymptotic Numerical Method (ANM). This framework was introduced in [4], and extended later for more general nonlinearities in [31]. A synthetic presentation is given in [32], where an extension to quasi-periodic solutions is built.

The HBM transforms the problem (1) (on $\mathbf{x}$ ) into a nonlinear algebraic system $\mathbf{R}(\mathbf{X}, \lambda)=0$ on Fourier coefficients of $\mathbf{x}$, its period and the continuation parameter $\lambda$. To use the ANM, this new system must verify the following assumption: there exist $\mathbf{C}_{0}, \mathbf{C}_{1} \in \mathbb{R}^{N}, \mathbf{L}_{0}, \mathbf{L}_{1}$ real $N \times N$ matrices, $\mathbf{Q}_{0}$ a bilinear application of $\mathbb{R}^{N} \times \mathbb{R}^{N} \rightarrow \mathbb{R}^{N}$, so that

$$
\mathbf{R}(\mathbf{X}, \lambda)=\mathbf{C}_{0}+\lambda \mathbf{C}_{1}+\mathbf{L}_{0} \mathbf{X}+\lambda \mathbf{L}_{1} \mathbf{X}+\mathbf{Q}_{0}(\mathbf{X}, \mathbf{X})
$$

As shown in [4], the system on Fourier unknowns can be obtained automatically and comply to the formalism (2), provided that the differential system (1) is first recast as follows:

$$
\mathbf{m} \dot{\mathbf{u}}=\mathbf{c}_{0}+\lambda \mathbf{c}_{1}+\mathbf{l}_{0} \mathbf{u}+\lambda \mathbf{l}_{1} \mathbf{u}+\mathbf{q}(\mathbf{u}, \mathbf{u}) .
$$

The vector $\mathbf{u}$ (length $N_{e q}$ ) is the vector of all variables, including auxiliary variables required to recast the nonlinearities (as shown in examples in [4]). In Eq. (3), $\mathbf{c}_{0}, \mathbf{c}_{1} \in \mathbb{R}^{N_{e q}}, \mathbf{m}, \mathbf{l}_{0}$ and $\mathbf{l}_{1}$ are $N_{e q} \times N_{e q}$ matrices, and $\mathbf{q}$ is a bilinear application $\mathbb{R}^{N_{e q}} \times \mathbb{R}^{N_{e q}} \rightarrow \mathbb{R}^{N_{e q}}$.

\subsection{Weyl fractional derivative and integral}

The investigation of periodic solutions requires using an appropriate definition for the fractional derivative. Let $\alpha$ be in ]0,1[ and $x$ a differentiable function. In this paper, we use the following definition of the fractional derivative of order $\alpha$ :

$$
D^{\alpha} x(t):=\frac{1}{\Gamma(1-\alpha)} \int_{-\infty}^{t}(t-\tau)^{-\alpha} \dot{x}(\tau) \mathrm{d} \tau
$$

with $\Gamma$ Euler's Gamma function. It is a generalized integral that makes sense for $x$ periodic. It is then linked to the Weyl fractional derivative [25] and therefore is called Weyl derivative in this paper. It becomes a 
standard integral if the support of $x$ has a lower bound. In particular, if $x$ is causal, then its Weyl derivative becomes its Caputo derivative, denoted here by $D_{C}^{\alpha}$ and commonly defined as:

$$
\text { for } x:\left[0,+\infty[\rightarrow \mathbb{R}, \forall \alpha \in] 0,1\left[, \forall t \geqslant 0, \quad D_{C}^{\alpha} x(t):=\frac{1}{\Gamma(1-\alpha)} \int_{0}^{t}(t-\tau)^{-\alpha} \dot{x}(\tau) \mathrm{d} \tau\right.\right.
$$

We focus on the Weyl derivative because using other definitions of the fractional derivative (for instance the Caputo derivative) in a fractional-differential system prohibits the existence of periodic solutions, as proved independently in [33] and [34]. The link between the solutions obtained with Caputo derivatives and the periodic solutions obtained with Weyl derivatives is explained in Section 2.3. When the Caputo definition is used, periodic cycles may be observed as attracting curves in a steady state, i.e. asymptotically; but they cannot be solutions of the system. Using the Weyl derivative, periodic cycles can be solutions of the system. As stated by Podlubny ([27], p. 80): "In such a case transient effects cannot be studied. However, taking [the lower bound for the integral] $a=-\infty$ is the necessary abstraction for the consideration of the steady-state processes, for example for studying the response of the fractional-order dynamic system to the periodic input signal, wave propagation in viscoelastic materials, etc". Section 2.3 explains how the current framework can be used to retrieve periodic cycles observed asymptotically with the Caputo derivative.

Following what Diethelm wrote on Caputo derivatives [30], the definition (4) can be extended to the order $\beta \in \mathbb{R}_{+} \backslash \mathbb{N}$ as:

$$
D^{\beta} x(t):=\frac{1}{\Gamma(\lceil\beta\rceil-\beta)} \int_{-\infty}^{t}(t-\tau)^{\lceil\beta\rceil-\beta-1} x^{(\lceil\beta\rceil)}(\tau) \mathrm{d} \tau,
$$

where $\lceil\cdot\rceil$ denotes the ceiling function. Setting $n:=\lceil\beta\rceil-1$ and $\alpha:=\beta-n \in] 0,1[$, one gets

$$
D^{\beta} x(t)=\frac{1}{\Gamma(1-\alpha)} \int_{-\infty}^{t}(t-\tau)^{-\alpha} x^{(n+1)}(\tau) \mathrm{d} \tau=D^{\alpha} x^{(n)} .
$$

Therefore, the methods given below for $\alpha \in] 0,1[$ can be applied to higher fractional orders $\beta$ by a reformulation of the system, setting $y:=x^{(n)}$, and studying $D^{\alpha} y$.

The fractional integral can be defined in a similar way. Called "left side Weyl integral" in [26], table 2.1, it is defined as

$$
\forall \alpha \in \mathbb{R}_{+} \backslash \mathbb{N}, \quad D^{-\alpha} x(t):=\frac{1}{\Gamma(\alpha)} \int_{-\infty}^{t}(t-\tau)^{\alpha-1} x(\tau) \mathrm{d} \tau .
$$

It is well-defined for periodic functions of zero mean and is linked to the Weyl derivative by the property:

$$
\forall \alpha \in] 0,1\left[, \quad D^{-\alpha} \dot{x}=D^{1-\alpha} x,\right.
$$

\subsection{Link with the Caputo derivative}

In the literature, a common definition of the fractional derivative is the Caputo derivative $D_{C}^{\alpha}$, recalled in def. (5). It can then be observed numerically for many systems that as $t$ tends to infinity, the solution seems to be attracted by a periodic function (a closed curve in the phase space). But as said in section 2.2, there exists no periodic solution for a system that contains a Caputo fractional operator. On a given example it is easy to prove by reduction to the absurd, using the fact that the Caputo derivative of a periodic function is not periodic. This fact is easily verified for a sine ([26], p. 39), and more generally the Caputo derivative of a $T$-periodic function is not $\tilde{T}$-periodic, for any $\tilde{T}$, with the trivial exception of the null function [35]. Then, if we consider the following example of a Van der Pol oscillator with a Caputo-derivative term:

$$
\forall t \geqslant 0, \quad \ddot{x}(t)-a_{1}\left(1-x(t)^{2}\right) \dot{x}(t)+\omega_{1}^{2} x(t)-\frac{\lambda}{\Gamma(1-\alpha)} \int_{0}^{t}(t-\tau)^{-\alpha} \dot{x}(\tau) \mathrm{d} \tau=0
$$

assuming there exists a $T$-periodic solution $x:[0,+\infty[\rightarrow \mathbb{R}$ is absurd: the standard derivatives $\dot{x}, \ddot{x}$ are $T$-periodic; and on the other hand the Caputo derivative $D_{C}^{\alpha} x$ is not $T$-periodic.

We chose a specific framework (Weyl derivative) to avoid this issue. Can it be used to find the periodic functions observed in the Caputo framework? In other words, when a periodic function seems to emerge as a steady-state asymptote in a system based on Caputo derivatives, is it a solution of the modified system where Caputo derivatives are replaced with Weyl derivatives, and $t$ is in $\mathbb{R}$ instead of $\mathbb{R}_{+}$? 
Let us assume that the system can be recast as an algebro-differential system

$$
M \dot{\mathbf{u}}=f(\mathbf{u})+g_{C}^{\alpha}(\mathbf{u}),
$$

thanks to auxiliary unknowns. In this formulation, $M$ is a real matrix possibly with zeros on the diagonal (some equations will then be purely algebraic), $f: \mathbb{R}^{N} \rightarrow \mathbb{R}^{N}$ is the nonlinear part of the system, and $g_{C}^{\alpha}$ is the operator containing Caputo derivatives. For instance,

$$
\ddot{x}-\left(1-x^{2}\right) D_{C}^{\alpha} x+x=0
$$

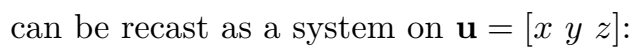

$$
\begin{aligned}
& \dot{x}=y \\
& \dot{y}=\left(1-x^{2}\right) z-x \\
& 0=z-D_{C}^{\alpha} x
\end{aligned}
$$

Then, let us suppose that there exists $\mathbf{u}_{0}, \mathbf{u}_{1}$ such that

$$
\mathbf{u}_{0} \text { periodic, } \quad\left\|\mathbf{u}_{1}(t)\right\| \underset{t \rightarrow+\infty}{\longrightarrow} 0, \quad\left\|\dot{\mathbf{u}}_{1}(t)\right\| \underset{t \rightarrow+\infty}{\longrightarrow} 0, \quad \mathbf{u}=\mathbf{u}_{0}+\mathbf{u}_{1} \text { is solution of system (11) }
$$

Then

$$
M \dot{\mathbf{u}}_{0}+M \dot{\mathbf{u}}_{1}=f\left(\mathbf{u}_{0}+\mathbf{u}_{1}\right)+g_{C}^{\alpha}\left(\mathbf{u}_{0}\right)+g_{C}^{\alpha}\left(\mathbf{u}_{1}\right)
$$

As $t$ tends to infinity, $M \dot{\mathbf{u}}_{1}$ tends to zero, and $f$ is smooth so $f\left(\mathbf{u}_{0}+\mathbf{u}_{1}\right)$ tends to $f\left(\mathbf{u}_{0}\right)$. We now deal with the limits of the Caputo derivatives. Let $T$ be the period of $\mathbf{u}_{0}$, and $k \in \mathbb{N}$. For each component $x_{0}$ of $\mathbf{u}_{0}$, $x_{0, \mathbb{R}}$ denotes the extension to a $T$-periodic function on $\mathbb{R}$, and we have

$$
\begin{aligned}
D_{C}^{\alpha} x_{0}(t+k T) & =\frac{1}{\Gamma(1-\alpha)} \int_{0}^{t+k T}(t+k T-\tau)^{-\alpha} \dot{x}_{0}(\tau) \mathrm{d} \tau \\
& =\frac{1}{\Gamma(1-\alpha)} \int_{-k T}^{t}(t-\sigma)^{-\alpha} \dot{x}_{0}(\sigma+k T) \mathrm{d} \sigma \\
& =\frac{1}{\Gamma(1-\alpha)} \int_{-k T}^{t}(t-\sigma)^{-\alpha} \dot{x}_{0, \mathbb{R}}(\sigma) \mathrm{d} \sigma \underset{k \rightarrow+\infty}{\longrightarrow} D^{\alpha} x_{0, \mathbb{R}}(t) .
\end{aligned}
$$

On the other hand, to conclude that $D_{C}^{\alpha} \mathbf{u}_{1}$ tends to zero, we add a sufficient condition of exponential convergence: for each component $x_{1}$ of $\mathbf{u}_{1}$,

$$
\exists C_{1}>1,\left|\dot{x}_{1}(t)\right|=\mathcal{O}\left(C_{1}^{-t}\right) \text { for } t \rightarrow+\infty, \quad \text { i.e. } \quad \exists C_{1}>1, \exists C_{0}, A_{1} \in \mathbb{R}_{+}, \forall t>A_{1},\left|\dot{x}_{1}(t)\right|<C_{0} C_{1}^{-t}
$$

Then, the following lemma (proved in AppendixA):

$$
\left[\exists C_{1}>1,\left|\dot{x}_{1}(t)\right|=\mathcal{O}\left(C_{1}^{-t}\right) \text { for } t \rightarrow+\infty\right] \Rightarrow\left|D_{C}^{\alpha} x_{1}(t)\right| \underset{t \rightarrow+\infty}{\longrightarrow} 0
$$

gives the result that $D_{C}^{\alpha} \mathbf{u}_{1}$ tends to zero. To summarize: the Caputo derivative of the "periodic part" $\mathbf{u}_{0}$ tends to the Weyl derivative of $\mathbf{u}_{0, \mathbb{R}}$, while under a sufficient condition on $\dot{\mathbf{u}}_{1}$ the Caputo derivative of the "residual" $\mathbf{u}_{1}$ tends to 0 . By taking the limit, $\mathbf{u}_{0, \mathbb{R}}$ verifies the system

$$
M \dot{\mathbf{u}}=f(\mathbf{u})+g^{\alpha}(\mathbf{u})
$$

where the fractional derivatives in $g^{\alpha}$ are now Weyl derivatives (and $t \in \mathbb{R}$ ).

\section{Harmonic Balance with Weyl derivative (HBW)}

\subsection{Expression of the Fourier coefficients}

When $D^{\alpha} x$ exists, its Fourier transform (denoted $\mathcal{F}$ ) can be computed and satisfies the property $([27]$, section 2.9):

$$
\mathcal{F}\left(D^{\alpha} x\right)(\xi)=(i \xi)^{\alpha} \mathcal{F}(x)(\xi) .
$$


As underlined in [26] (section 2.11.1), "if a periodic function is defined by its Fourier series it can be fractionally derivated term by term and the derivative is also periodic with the same period". When expressed with complex Fourier coefficients

$$
x(t)=\sum_{k \in \mathbb{Z}} x_{k} e^{i k \omega t}
$$

the property (22) reads:

$$
D^{\alpha} x(t)=\sum_{k \in \mathbb{Z}}(i k \omega)^{\alpha} x_{k} e^{i k \omega t}=\sum_{k \in \mathbb{Z}} \exp \left(i \frac{\pi}{2} \alpha\right)(k \omega)^{\alpha} x_{k} e^{i k \omega t}
$$

that is, with real Fourier coefficients:

$$
\begin{gathered}
x(t)=x_{0}+\sum_{k>0} x_{c k} \cos (k \omega t)+x_{s k} \sin (k \omega t), \\
D^{\alpha} x(t)=\sum_{k>0}(k \omega)^{\alpha}\left(\cos \left(\frac{\pi \alpha}{2}\right) x_{c k}+\sin \left(\frac{\pi \alpha}{2}\right) x_{s k}\right) \cos (k \omega t) \\
+(k \omega)^{\alpha}\left(-\sin \left(\frac{\pi \alpha}{2}\right) x_{c k}+\cos \left(\frac{\pi \alpha}{2}\right) x_{s k}\right) \sin (k \omega t) .
\end{gathered}
$$

Although the definition of $D^{\alpha}$ is restricted to non-integers orders, Eq. (26) gives a smooth expression of coefficients with respect to $\alpha$ i.e. without singularity at $\alpha \in \mathbb{N}$. For $x$ periodic, when $\alpha$ tends to $0, D^{\alpha} x$ tends to $x$; when $\alpha$ tends to $1, D^{\alpha} x$ tends to $\dot{x}$.

For numerical applications, both $x$ and $D^{\alpha} x$ are now decomposed on $H$ harmonics: in the Eqs. (25), (26), the sums are finite (for $k$ between 1 and $H$ ).

\subsection{A simple implementation: constant rational order}

In a first case, let us assume $\alpha \in \mathbb{Q}$. After defining an auxiliary unknown

$$
\Omega=\omega^{\alpha}
$$

the expression (26) complies with the ANM formalism of Eq. (2) since it only contains products of unknowns $\left(\Omega\right.$ and $x_{c k}$ or $x_{s k}$ ). Then, $\alpha$ being a rational $p / q$, the definition $(27)$ can be transformed into a small quadratic system. The simplest example, which is useful for heat conduction for instance, is $\alpha=1 / 2$. No transformation is needed because $\Omega^{2}=\omega$ is a quadratical definition. This simple case will be used as a first example in Section 5. If for example $\alpha=3 / 5$, the quadratical definitions

$$
\rho_{1}=\Omega^{2}, \quad \rho_{2}=\rho_{1}^{2}, \quad \rho_{3}=\omega^{2}, \quad \rho_{2} \Omega=\omega \rho_{3}
$$

yield $\Omega^{5}=\omega^{3}$ i.e. $\Omega=\omega^{3 / 5}$. One can see the drawbacks of this approach:

1. if the reduced fraction $\alpha=p / q$ calls for large integers $p, q$, then $\omega$ is raised to a large power, which leads to a loss of accuracy.

2. this is not applicable to $\alpha$ irrational. Moreover, using an approximate rational value $p / q \simeq \alpha$ leads again to the first point ( $p, q$ large exponents) when the precision of this rational approximation is increased.

3. this is not applicable if $\alpha$ is the continuation parameter instead of a constant.

This motivates a more general implementation.

\subsection{Implementation: general case}

We present the Harmonic Balance with Weyl derivative (HBW) in the most general case, where $\alpha$ is the continuation parameter (not a constant), and this case will be illustrated in Section 5 . Then we explain how any constant $\alpha$ can be handled, which solves the two problematic cases outlined above $(\alpha=p / q$ and $p, q \gg 1$; $\alpha$ irrational). 
To perform a ANM continuation with respect to the derivation order $\alpha$, the quantities in Eq. (26) that need to be defined in a quadratical manner are of the form

$$
(k \omega)^{\alpha} \cos \left(\frac{\pi \alpha}{2}\right) x_{c k} .
$$

The nonlinear functions in Eq. (29) (cos, sin, exp) satisfy linear, first-order differential systems. These functions are applied to Fourier unknowns $(\omega)$ or the continuation parameter $\alpha$, that is to say, to unknowns of the ANM system. Thus reframed, this issue is solved by differentiation of the equations with respect to the path parameter of the ANM continuation, as presented in [31]. Details of the equations and auxiliary unknowns are given in AppendixB. We give here the framework (presented in [31]) and one example. The formalism (2) enables the ANM to obtain linear systems on the Taylor coefficients of the unknowns. If $\mathbf{X}$ denotes the vector of ANM unknowns, and $\mathrm{d} \mathbf{X}$ its differentiation with respect to the path parameter $a$, linear systems (on the Taylor coefficients of $\mathbf{X}$ ) can also be obtained if some equations are written in a system of so-called differentiated equations:

$$
\mathbf{L}_{h}(\mathrm{~d} \mathbf{X})=\mathbf{Q}_{h}(\mathbf{X}, \mathrm{d} \mathbf{X})
$$

where $\mathbf{L}_{h}$ is linear, $\mathbf{Q}_{h}$ is bilinear. To give an example, if we set the auxiliary unknown

$$
A_{k}:=k^{\alpha} \quad(2 \leqslant k \leqslant H)
$$

then $A_{k}$ can be defined by a differentiated equation:

$$
\forall k \in \llbracket 2, H \rrbracket, \quad \mathrm{d} A_{k}=\ln (k) A_{k} \mathrm{~d} \alpha
$$

The reader is referred to AppendixB for more details. Now, if $\alpha$ is an arbitrary constant, in Eq. (29), the only term that must be recast as an auxiliary unknown is $\omega^{\alpha}=\exp (\alpha \ln \omega)$ (the other terms are constants or a Fourier coefficient), and this is done with differentiated equations, as shown in the appendix.

\section{Diffusive representation and approximation}

To confirm the results when the fractional order $\alpha$ is constant, we now introduce a different method. Its two steps are:

1. the diffusive representation, which is an exact reformulation of a fractional derivative or integral. It is now well established for Caputo fractional operators [30] and we will carry out very similar computations for Weyl operators;

2. the diffusive approximation, where the previous representation is approached.

Let us start with the fractional derivative. By defining for $\theta \in[0,+\infty[$ :

$$
\Phi(\theta, t):=\frac{2 \sin (\pi \alpha)}{\pi} \theta^{2 \alpha-1} e^{-t \theta^{2}} \int_{-\infty}^{t} e^{\tau \theta^{2}} \dot{x}(\tau) \mathrm{d} \tau,
$$

one verifies that (details of the calculation are in AppendixC.1):

$$
D^{\alpha} x(t)=\int_{0}^{\infty} \Phi(\theta, t) \mathrm{d} \theta
$$

which is called the diffusive representation of $D^{\alpha} x$. Then, the integral can be approached by a quadrature formula. For an appropriate choice of $n_{\phi}$ weights $\mu_{\ell}$ and nodes $\theta_{\ell}$, we define $\Phi_{\ell}:=\Phi\left(\theta_{\ell}, \cdot\right)$, and $D^{\alpha} x$ is replaced by $\sum_{\ell=1}^{n_{\phi}} \mu_{\ell} \Phi_{\ell}$. We will call this sum the diffusive approximation. Each function $\Phi_{\ell}$ can be defined by the differential system

$$
\begin{aligned}
\dot{\Phi}_{\ell} & =-\theta_{\ell}^{2} \Phi_{\ell}+\frac{2 \sin (\pi \alpha)}{\pi} \theta_{\ell}^{2 \alpha-1} \dot{x} \\
\Phi_{\ell}(0) & =\frac{2 \sin (\pi \alpha)}{\pi} \theta_{\ell}^{2 \alpha-1} \int_{-\infty}^{0} e^{\tau \theta_{\ell}^{2}} \dot{x}(\tau) \mathrm{d} \tau .
\end{aligned}
$$


Thus a system with Weyl derivative of constant order $\alpha$ can be approached with a differential system. Its periodic solutions can then be continued with different continuation methods; our choice will be the standard ANM-HBM. The only difficulty is that Eq. (35b) must be taken into account for each function $\Phi_{\ell}$. We show in AppendixD that Eq. (35b) can be rewritten as a quadratical system on the Fourier unknowns, and therefore it can comply with the ANM formalism. The method used here to obtain the quadrature nodes $\theta_{\ell}$ and weights $\mu_{\ell}$ is briefly recalled in AppendixC.2. In the same appendix, the similar procedure of diffusive approximation of fractional integrals is given.

\section{Examples of continuation}

Two examples are now presented to illustrate the method. For the sake of simplicity they are scalar equations (one oscillator similar to the Van der Pol oscillator), but there is no restriction on the dimension of the differential fractional system: the method can be applied to multiple coupled degrees of freedom. Also, the nonlinearity in Van der Pol oscillator is polynomial, but as explained in 2.1, the ANM-HBM framework can deal with other nonlinearities as well. Finally, these examples are intended to illustrate the numerical method, which is the main focus of this paper. In the spirit of previous studies [36-39], the authors chose fractional Van der Pol oscillators; they are mathematical illustrations, not physical models.

\subsection{Continuation with constant rational order}

In this section, we continue periodic solutions of

$$
\ddot{x}-a_{1}\left(1-x^{2}\right) \dot{x}+\omega_{1}^{2} x-\lambda D^{1 / 2} x=0
$$

with respect to the parameter $\lambda$. Notice that in this example the derivative order is constant and equal to a rational value, $\alpha=\frac{1}{2}$. Parameters values are: $\omega_{1}=200 \pi, a_{1}=2 \omega_{1}$. By adding three auxiliary variables, Eq. (36) is recast in a system similar to (3), setting aside the fractional operator:

$$
\begin{aligned}
\dot{x} & =\omega_{1} x_{2} \\
\dot{x}_{2} & =a_{1} x_{2}-\omega_{1} x+\frac{\lambda}{\omega_{1}} x_{4}-a_{1} x_{2} x_{3} \\
0 & =x_{3}-x^{2} \\
0 & =x_{4}-D^{1 / 2} x
\end{aligned}
$$

The results will be confirmed using a diffusive approximation (section 4). The initial condition of function $\Phi$ is slightly easier to implement for fractional integrals (see AppendixD). Eq. (9) shows that Eq. (36) is equivalent to:

$$
\ddot{x}-a_{1}\left(1-x^{2}\right) \dot{x}+\omega_{1}^{2} x-\lambda D^{-1 / 2} \dot{x}=0
$$

Then, thanks to the diffusive approximation of the fractional integral (system (C.10)), Eq. (38) is approached by the system:

$$
\begin{aligned}
\dot{x} & =\omega_{1} x_{2} \\
\dot{x}_{2} & =a_{1} x_{2}-\omega_{1} x+\frac{\lambda}{\omega_{1}} \sum_{\ell=1}^{n_{\phi}} \mu_{\ell} x_{2+\ell}-a_{1} x_{2} x_{3+n_{\phi}} \\
\forall \ell \in \llbracket 1, n_{\phi} \rrbracket, \quad \dot{x}_{2+\ell} & =-\theta_{\ell}^{2} x_{2+\ell}+\frac{2 \omega_{1}}{\pi} x_{2} \\
0 & =x_{3+n_{\phi}}-x^{2}
\end{aligned}
$$

Values of nodes $\theta_{\ell}$ and weights $\mu_{\ell}$ (according to the optimization procedure given in AppendixC.2) are given in table 1 for the choice $n_{\phi}=6$.

To study periodic solutions of the first system (37), we use the Harmonic Balance with Weyl derivative (section 3.2). For the second system (39), the usual Harmonic Balance from [4] is applied. The adimensional angular frequency of the solution $\omega / \omega_{1}$ and the peak-to-peak amplitude of $x$ are represented in Fig. 1. Using 


\begin{tabular}{|l|l|l|l|l|l|l|}
\hline$\theta_{\ell}$ & 5.748987 & 17.913444 & 41.612967 & 95.501586 & 221.848952 & 691.235767 \\
\hline$\mu_{\ell}$ & 10.965871 & 15.622528 & 34.604044 & 79.417073 & 193.468069 & 1318.469721 \\
\hline
\end{tabular}

Table 1: Values of nodes $\theta_{\ell}$ and weights $\mu_{\ell}$ for $n_{\phi}=6$, used in Eq. (39).

the diffusive approximation gives access to standard techniques of stability analysis. Floquet exponents can be computed through Hill's method, which is efficiently coupled with the ANM-HBM [40]. Thanks to this indication given by the diffusive approximation we conjecture that the periodic branch is stable. The periodic branch and the trivial stationary branch $(x=0)$ meet at a bifurcation which is now commented.

For the diffusive approximation, this is a Hopf bifurcation. It occurs at the first crossing of the imaginary axis by a pair of eigenvalues of the Jacobian of the differential system $((39 a)-(39 c))$, evaluated at the (null) stationary solution, which is the matrix of size $2+n_{\phi}$

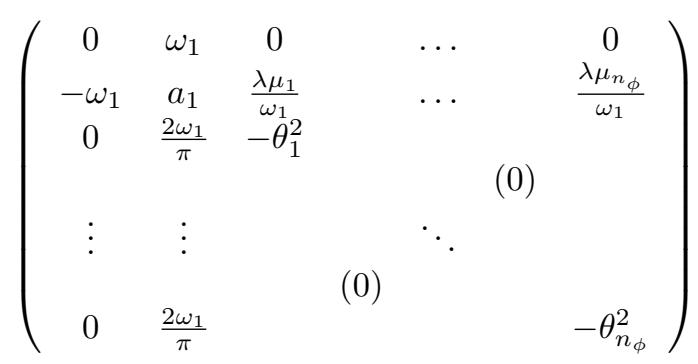

Moreover, the periodic solution (obtained with the HBW) has some remarkable properties in the vicinity of the bifurcation: a good approximation can be obtained with few harmonics and there is a square-root growth of the amplitude with respect to $\lambda$. Based on these properties and the analysis provided by the diffusive approximation, we conjecture that the bifurcation (for the original fractional system of Eq. (36)) can be called a Hopf bifurcation and that an appropriate framework could be theorized to include this type of bifurcation. To our knowledge, the main stability results available in the literature (such as [41]) are given in the framework of causal systems with Caputo derivative. In this framework the "Hopf bifurcation" describes the emergence of asymptotically stable limit cycles (see for instance [42]), which unfortunately lacks generality in two ways: as recalled in section 2.2 these cycles are not solutions; and inverse bifurcations, where the emerging limit cycle is unstable, can not be described.

Under the conjecture that the bifurcation is a typical Hopf bifurcation, in its vicinity we can approach $x$ with one harmonic. For an appropriate phase, and given that the stationary branch is null, it means that $x$ can be approached by $x(t)=x_{1} \cos (\omega t)$. Then, the bifurcation can be found as follows: substitute $x_{1} \cos (\omega t)$ in Eq. (36), perform the harmonic balance on the first cosine and sine, assume the solution $x$ is null, take the limit $x_{1} \rightarrow 0$. We find that at the bifurcation, $\omega$ and the continuation parameter $\lambda$ verify the nonlinear system:

$$
\begin{aligned}
& -\omega^{2}+\omega_{1}^{2}-\cos \left(\frac{\pi}{4}\right) \lambda \sqrt{\omega}=0 \\
& a_{1} \omega-\lambda \sin \left(\frac{\pi}{4}\right) \sqrt{\omega}=0
\end{aligned}
$$

For the values of parameters chosen here, the solutions of this system are:

$$
\frac{\omega_{\text {theo }}}{\omega_{1}} \simeq 2.41, \quad \lambda_{\text {theo }} \simeq-6.92 \times 10^{4}
$$

For $n_{\phi}=6$, the values of $\omega, \lambda$ at the Hopf bifurcation for the diffusive approximation are in good agreement with the solutions of (42) : the relative error on $\lambda$ (respectively, on $\omega$ ) is $9 \times 10^{-3}$ (resp. $5 \times 10^{-3}$ ).

For $\lambda=0$ the fractional term of the system is not present, which means that both methods necessarily agree there. It can also be used as a starting point for the continuation. For $\lambda>4 \times 10^{4}$ the continuation becomes increasingly expensive in computation time. The reason is that while the period increases, for an increasingly long portion of the period $\dot{x}$ is near 0 . This needs more harmonics to be accurately approached, and therefore computation time per step increases.

Examples of solutions, extracted from the bifurcation diagram, are given in Fig. 2. 
a)

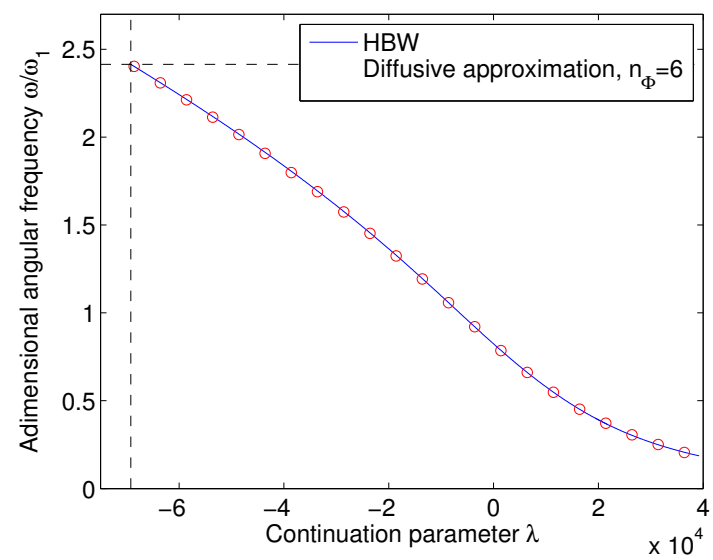

b)

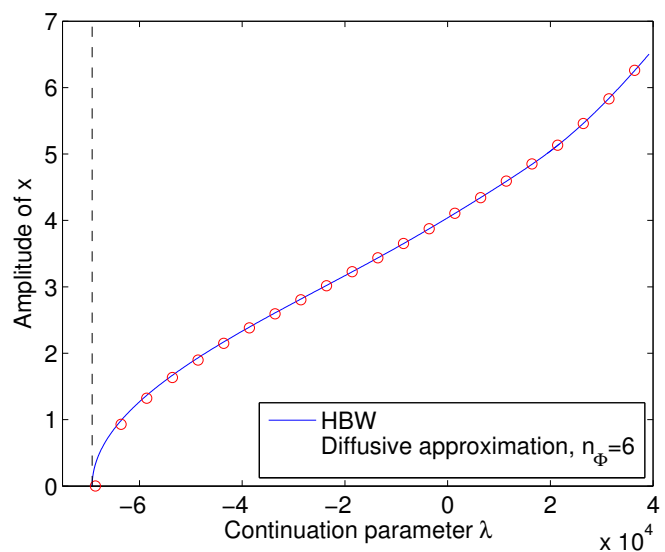

Figure 1: Continuation of periodic solutions of Eq. (36). a) Adimensional angular frequency $\omega / \omega_{1}$ vs the continuation parameter $\lambda$. Harmonic Balance with Weyl derivative (HBW) in blue solid line; diffusive approximation with $n_{\phi}=6$, red circles. b) Peak-topeak amplitude of $x$ vs $\lambda$. The values $\omega_{\text {theo }}, \lambda_{\text {theo }}$ are shown in black.

a)

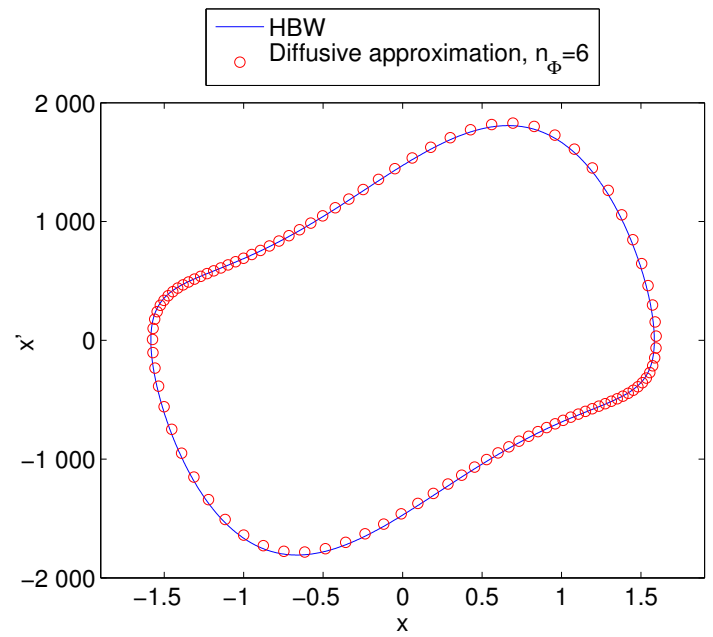

b)

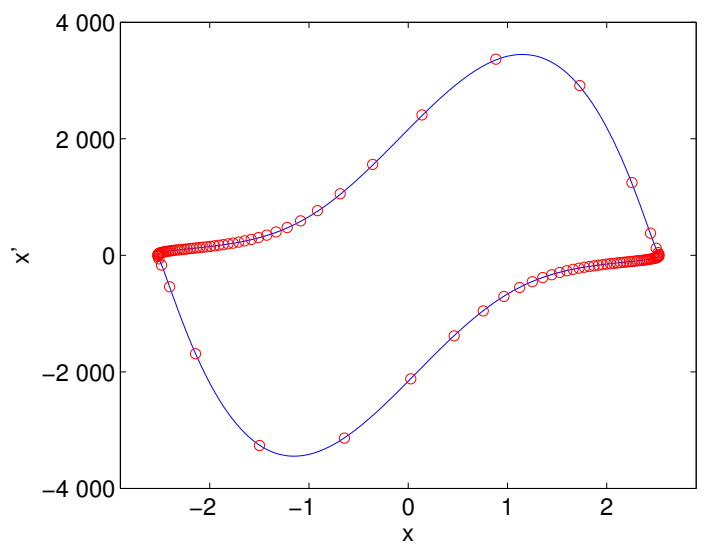

Figure 2: Examples of phase diagrams $(x, \dot{x})$ of periodic solutions of Eq. (36). a) $\lambda=-2 \times 10^{4}$. HBW (blue, solid); diffusive approximation with $n_{\phi}=6$ (red circles). b) $\lambda=2 \times 10^{4}$. 


\subsection{Continuation with respect to the derivation order}

In this section, the periodic solutions of

$$
\ddot{x}-a_{1}\left(1-x^{2}\right) \dot{x}+\omega_{1}^{2} x+b_{1} D^{\alpha} x=0
$$

are continued with respect to the order of the fractional derivative (that is to say, in the ANM formalism (2), $\lambda=\alpha)$. Parameters values are: $\omega_{1}=200 \pi, a_{1}=2 \omega_{1}, b_{1}=5 \omega_{1}$. The system can be written

$$
\begin{aligned}
\dot{x} & =\omega_{1} x_{2} \\
\dot{x}_{2} & =a_{1} x_{2}-\omega_{1} x-\frac{b_{1}}{\omega_{1}} D^{\alpha} x-a_{1} x_{2} x_{3} \\
0 & =x_{3}-x^{2}
\end{aligned}
$$

and periodic solutions are continued as presented in section 3.3. The adimensional angular frequency of the solution $\omega / \omega_{1}$ and the peak-to-peak amplitude of $x$ are represented in Fig. 3. The periodic branch and the trivial stationary branch $(x=0)$ meet at a bifurcation. Again, the periodic solution in the vicinity of the bifurcation can be approached with few harmonics, and its amplitude grows as the square root of $\lambda$. We conjecture that it can be called a Hopf bifurcation and that an appropriate framework could be theorized to include this type of bifurcation. Closing this theoretical gap would justify the same conjecture as before: in the vicinity of the bifurcation the solution is well approached with one harmonic, and similarly to system (41) we obtain that at the bifurcation, the pulsation $\omega$ and the order $\alpha$ verify the following nonlinear system :

$$
\begin{aligned}
& -\omega^{2}+\omega_{1}^{2}+b_{1} \omega^{\alpha} \cos \left(\frac{\pi \alpha}{2}\right)=0 \\
& a_{1} \omega-b_{1} \omega^{\alpha} \sin \left(\frac{\pi \alpha}{2}\right)=0
\end{aligned}
$$

For the values of parameters chosen here, the solutions are: $\frac{\omega_{\text {theo }}}{\omega_{1}} \simeq 1.24, \alpha_{\text {theo }} \simeq 0.866$.

For $\alpha$ crossing integer values, the periodic solutions of Eq. (43) can be found by replacing $D^{\alpha} x$ with a standard derivation or integration. The simplest case here is for $\alpha=0$, where the branch of periodic solutions passes through the periodic solution of

$$
\ddot{x}-a_{1}\left(1-x^{2}\right) \dot{x}+\omega_{1}^{2} x+b_{1} x=0
$$

This remark may be used to obtain a starting point for the continuation. Another way to obtain a starting point can be the previous study: Eqs. (36) and (43) are the same for appropriate parameters $\left(\lambda=-5 \omega_{1}\right.$ in the first one, $\alpha=\frac{1}{2}$ in the second one).

\section{Conclusion}

We have shown that the correct framework for defining periodic solutions of fractional systems is the Weyl derivative, whose link with the usual Caputo derivative has been analyzed. Within this framework, two methods based on the use of HBM and ANM have been extended to fractional systems. The examples of application proposed here have illustrated the complementary qualities of these approaches, on an academic case. However, these approaches open the way to the continuation of periodic solutions of many physical systems modeled by fractional derivatives.

This work opens perspectives for research on periodic solutions of systems with fractional derivatives. For instance, bifurcations connecting the periodic and stationary branches were found in the numerical experiments, presenting a great similarity with the Hopf bifurcation in differential systems. However, the theoretical framework in the non-standard case seems to be currently missing. Similar questions arise concerning the stability of periodic solutions of fractional systems. We hope that the numerical method developed here can stimulate new developments on these theoretical issues. 
a)

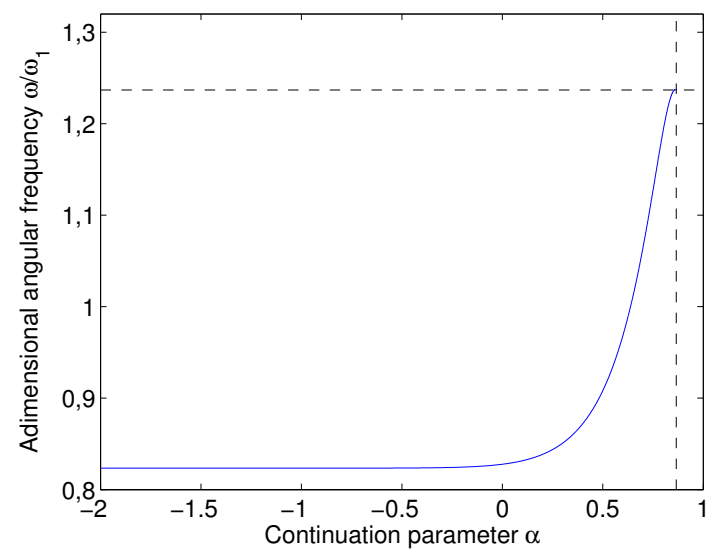

b)

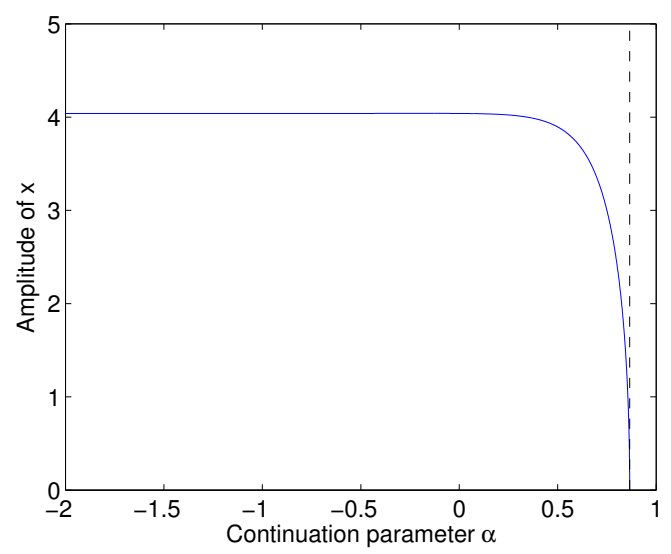

Figure 3: Continuation of periodic solutions of Eq. (43). a) Adimensional angular frequency $\frac{\omega}{\omega_{1}}$ vs the continuation parameter $\alpha$. b) Peak-to-peak amplitude of $x$ vs $\alpha$. The values $\omega_{\text {theo }}, \alpha_{\text {theo }}$ are shown in black.

\section{Acknowledgements}

This work has been carried out in the framework of the Labex MEC (ANR-10-LABX-0092) and of the A*MIDEX project (ANR-11-IDEX-0001-02), funded by the Investissements d'Avenir French Government program managed by the French National Research Agency (ANR).

\section{AppendixA. Lemma}

In this section we prove the implication (20). The hypothesis on $\dot{x}_{1}$ is

$$
\exists C_{1}>1,\left|\dot{x}_{1}(t)\right|=\mathcal{O}\left(C_{1}^{-t}\right) \text { for } t \rightarrow+\infty, \quad \text { i.e. } \quad \exists C_{1}>1, \exists C_{0}, A_{1} \in \mathbb{R}_{+}, \forall t>A_{1},\left|\dot{x}_{1}(t)\right|<C_{0} C_{1}^{-t}
$$

Let $\varepsilon>0$. Let $\delta>\frac{1}{\alpha}>1$. Since

$$
t^{1-\delta} \underset{t \rightarrow+\infty}{\longrightarrow} 0, \quad t^{1-\alpha \delta} \underset{t \rightarrow+\infty}{\longrightarrow} 0, \quad C_{1}^{-t} t^{\delta(1-\alpha)} \underset{t \rightarrow+\infty}{\longrightarrow} 0
$$

let $A>A_{1}$ be such that for all $B>A$,

$$
\begin{aligned}
& (1-\alpha) B^{1-\delta}<1 \\
& \sup _{\tau \in \mathbb{R}_{+}}\left|\dot{x}_{1}(\tau)\right|\left(e^{1}-1\right) B^{1-\alpha \delta}<\frac{\varepsilon}{2} \\
& \frac{C_{0}}{1-\alpha} C_{1}^{-B} B^{\delta(1-\alpha)}<\frac{\varepsilon}{2}
\end{aligned}
$$

Then, for all $t>A+A^{\delta}, \exists B>A$ such that $t=B+B^{\delta}$, and one has

$$
\begin{aligned}
\left|\Gamma(1-\alpha) D_{C}^{\alpha} x_{1}(t)\right| & =\left|\int_{0}^{B+B^{\delta}}\left(B+B^{\delta}-\tau\right)^{-\alpha} \dot{x}_{1}(\tau) \mathrm{d} \tau\right| \\
& \leqslant \underbrace{\left|\int_{0}^{B}\left(B+B^{\delta}-\tau\right)^{-\alpha} \dot{x}_{1}(\tau) \mathrm{d} \tau\right|}_{I_{1}}+\underbrace{\left|\int_{B}^{B+B^{\delta}}\left(B+B^{\delta}-\tau\right)^{-\alpha} \dot{x}_{1}(\tau) \mathrm{d} \tau\right|}_{I_{2}}
\end{aligned}
$$


Now the two terms $I_{1}$ and $I_{2}$ will be upper bounded. For the first term:

$$
\begin{aligned}
I_{1} & \leqslant \sup _{\tau \in[0, B]}\left|\dot{x}_{1}(\tau)\right| \int_{0}^{B}\left(B+B^{\delta}-\tau\right)^{-\alpha} \mathrm{d} \tau \\
& \leqslant \sup _{\tau \in \mathbb{R}_{+}}\left|\dot{x}_{1}(\tau)\right|\left[-\frac{\left(B+B^{\delta}-\tau\right)^{1-\alpha}}{1-\alpha}\right]_{\tau=0}^{B} \\
& \leqslant \sup _{\tau \in \mathbb{R}_{+}}\left|\dot{x}_{1}(\tau)\right| \frac{\left(B+B^{\delta}\right)^{1-\alpha}-\left(B^{\delta}\right)^{1-\alpha}}{1-\alpha}
\end{aligned}
$$

Since

$$
\left(B+B^{\delta}\right)^{1-\alpha}=\exp \left((1-\alpha) \ln \left(B^{\delta}\left(1+B^{1-\delta}\right)\right)\right) \leqslant \exp \left((1-\alpha)\left[\delta \ln B+B^{1-\delta}\right]\right)=B^{\delta(1-\alpha)} \exp \left((1-\alpha) B^{1-\delta}\right)
$$

one has

$$
\left(B+B^{\delta}\right)^{1-\alpha}-B^{\delta(1-\alpha)} \leqslant B^{\delta(1-\alpha)}\left(\exp \left((1-\alpha) B^{1-\delta}\right)-1\right)
$$

The exponential function is convex, so

$$
\forall x \in[0,1], \quad e^{x} \leqslant\left(e^{1}-1\right) x+1, \quad \text { i.e. } \quad e^{x}-1 \leqslant\left(e^{1}-1\right) x
$$

By hypothesis (A.3a), $(1-\alpha) B^{1-\delta} \in[0,1]$, so Eqs. (A.10) and (A.11) give

$$
\left(B+B^{\delta}\right)^{1-\alpha}-B^{\delta(1-\alpha)} \leqslant B^{\delta(1-\alpha)}\left(e^{1}-1\right)(1-\alpha) B^{1-\delta}=\left(e^{1}-1\right)(1-\alpha) B^{1-\alpha \delta}
$$

Therefore by hypothesis (A.3b),

$$
\sup _{\tau \in \mathbb{R}_{+}}\left|\dot{x}_{1}(\tau)\right| \frac{\left(B+B^{\delta}\right)^{1-\alpha}-\left(B^{\delta}\right)^{1-\alpha}}{1-\alpha}<\frac{\varepsilon}{2}
$$

For the second term $I_{2}$ :

$$
\begin{aligned}
I_{2} & \leqslant \sup _{\tau>B}\left|\dot{x}_{1}(\tau)\right| \int_{B}^{B+B^{\delta}}\left(B+B^{\delta}-\tau\right)^{-\alpha} \mathrm{d} \tau \\
& \leqslant C_{0} C_{1}^{-B}\left[-\frac{\left(B+B^{\delta}-\tau\right)^{1-\alpha}}{1-\alpha}\right]_{\tau=B}^{B+B^{\delta}} \quad \text { (hypothesis (A.1)) } \\
& \leqslant C_{0} C_{1}^{-B} \frac{\left(B^{\delta}\right)^{1-\alpha}}{1-\alpha} \\
& \leqslant \frac{\varepsilon}{2} \quad(\text { hypothesis (A.3c)) }
\end{aligned}
$$

Remark. The exponential convergence, which we observed numerically, is not an excessively demanding condition, or in contradiction with the slow convergence typically observed for fractional systems (the constant $C_{1}$ in Eq. (A.1) can be close to 1). The conclusion that $D_{C}^{\alpha} x_{1}$ tends to zero may hold for a slower convergence of $\dot{x}_{1}$ and specific values of $\alpha$. For instance, to accomodate the asymptotical behaviour proved in [43] for some fractional systems, we note that the proof above works if $\dot{x}_{1}(t)=\mathcal{O}\left(t^{-\gamma}\right)(\gamma>0)$ and if the hypothesis (A.3c) is replaced with: $t^{-\gamma+\delta(1-\alpha)} \underset{t \rightarrow+\infty}{\longrightarrow} 0$, i.e. $-\gamma+\delta(1-\alpha)<0$. Then, if $\frac{1}{\alpha}<\frac{\gamma}{1-\alpha}$, choosing $\delta$ in $] \frac{1}{\alpha}, \frac{\gamma}{1-\alpha}[$ in the proof above is sufficient to meet all hypotheses. 


\section{AppendixB. ANM equations for the continuation with respect to the fractional order}

In this appendix, the equations used in the ANM formalism for the continuation with respect to $\alpha$ are given. Along the standard HBM equations, two types of equations will be added: quadratic equations, and so-called differentiated equations.

The aim is to have a quadratic formalism for expressions such as Eq. (29), recalled here:

$$
(k \omega)^{\alpha} \cos \left(\frac{\pi \alpha}{2}\right) x_{c k}
$$

which can be subdivided in smaller problems: if

$$
A_{k}:=k^{\alpha}(2 \leqslant k \leqslant H), \quad O_{L}:=\omega^{\alpha}, \quad c:=\cos \left(\frac{\pi \alpha}{2}\right), \quad s:=\sin \left(\frac{\pi \alpha}{2}\right)
$$

were defined as auxiliary unknowns, then an expression such as (29) would become a product of four unknowns, which is tractable in the quadratic formalism (provided a few more auxiliary unknowns). We set

$$
\Omega:=\ln \omega
$$

and then the $A_{k}, \Omega, c$ and $s$ can be defined by differentiated equations :

$$
\begin{gathered}
\omega=\exp \Omega \quad \text { so } \quad \mathrm{d} \omega=\omega \mathrm{d} \Omega \\
\forall k \in \llbracket 2, H \rrbracket, \quad \mathrm{d} A_{k}=\ln (k) A_{k} \mathrm{~d} \alpha \\
\mathrm{d} c=-\frac{\pi}{2} s \mathrm{~d} \alpha, \quad \mathrm{d} s=\frac{\pi}{2} c \mathrm{~d} \alpha
\end{gathered}
$$

Then, we add the quadratical equation

$$
\rho:=\alpha \Omega
$$

yielding the differentiated definition of $O_{L}=\exp (\alpha \Omega)$ :

$$
\mathrm{d} O_{L}=O_{L} \mathrm{~d} \rho
$$

Then some quadratic equations are added:

$$
\begin{aligned}
O_{C} & =O_{L} c \\
O_{S} & =O_{L} s \\
B_{k} & =A_{k} O_{C}, \quad \forall k \in \llbracket 2, H \rrbracket \\
C_{k} & =A_{k} O_{S}, \quad \forall k \in \llbracket 2, H \rrbracket
\end{aligned}
$$

In summary, $3+2(H-1)$ quadratic equations were added (Eqs. (B.5), (B.7)); and $4+(H-1)$ differentiated equations (Eqs. (B.2), (B.3), (B.4), (B.6)). The vector of unknowns, besides the Fourier coefficients of variables, the pulsation and the continuation parameter, now contains $3 H+4$ extra unknowns.

\section{AppendixC. Diffusive representation and approximation}

\section{AppendixC.1. Diffusive representation}

Firstly we show a proof of Eq. (34).

Proof. One recalls that

$$
\Gamma(\alpha):=\int_{0}^{\infty} e^{-z} z^{\alpha-1} \mathrm{~d} z, \quad \Gamma(\alpha) \Gamma(1-\alpha)=\frac{\pi}{\sin (\pi \alpha)}
$$


Similarly to the computations for the Caputo fractional derivative [30] :

$$
\begin{aligned}
D^{\alpha} x(t) & =\frac{\Gamma(\alpha)}{\Gamma(\alpha) \Gamma(1-\alpha)} \int_{-\infty}^{t}(t-\tau)^{-\alpha} \dot{x}(\tau) \mathrm{d} \tau \\
& =\frac{\sin (\pi \alpha)}{\pi} \int_{z=0}^{\infty} e^{-z} z^{\alpha-1} \mathrm{~d} z \int_{\tau=-\infty}^{t}(t-\tau)^{-\alpha} \dot{x}(\tau) \mathrm{d} \tau \\
& =\frac{\sin (\pi \alpha)}{\pi} \int_{z=0}^{\infty} \int_{\tau=-\infty}^{t} e^{-z}\left(\frac{z}{t-\tau}\right)^{\alpha} \frac{\dot{x}(\tau)}{z} \mathrm{~d} \tau \mathrm{d} z \\
& =\frac{2 \sin (\pi \alpha)}{\pi} \int_{\theta=0}^{\infty} \int_{\tau=-\infty}^{t} e^{-(t-\tau) \theta^{2} \theta^{2 \alpha-1} \dot{x}(\tau) \mathrm{d} \tau \mathrm{d} \theta} \\
& =\int_{\theta=0}^{\infty} \frac{2 \sin (\pi \alpha)}{\pi} \theta^{2 \alpha-1} e^{-t \theta^{2}} \int_{\tau=-\infty}^{t} e^{\tau \theta^{2}} \dot{x}(\tau) \mathrm{d} \tau \mathrm{d} \theta \\
& =\int_{\theta=0}^{\infty} \Phi(\theta, t) \mathrm{d} \theta
\end{aligned}
$$

according to the definition (33).

For fractional integrals, by defining for $\theta \in[0,+\infty[$ :

$$
\Phi^{-\alpha}(\theta, t):=\frac{2 \sin (\pi \alpha)}{\pi} \theta^{1-2 \alpha} e^{-t \theta^{2}} \int_{-\infty}^{t} e^{\tau \theta^{2}} x(\tau) \mathrm{d} \tau
$$

one obtains similarly

$$
D^{-\alpha} x(t)=\int_{0}^{\infty} \Phi^{-\alpha}(\theta, t) \mathrm{d} \theta
$$

\section{AppendixC.2. Diffusive approximation}

The diffusive representations (either Eq. (34) or (C.9)) can be approached by quadrature formulas. For a given node $\theta_{\ell}, \Phi_{\ell}$ denotes $\Phi\left(\theta_{\ell}, \cdot\right)$. The diffusive representation is approached by a sum $\sum_{\ell=1}^{n_{\phi}} \mu_{\ell} \Phi_{\ell}$. Each function $\Phi_{\ell}$ is solution of a differential system. For the fractional derivative, it is the system (35); for the fractional integral, it is the following system:

$$
\begin{aligned}
\dot{\Phi}_{\ell}^{-\alpha} & =-\theta_{\ell}^{2} \Phi_{\ell}^{-\alpha}+\frac{2 \sin (\pi \alpha)}{\pi} \theta_{\ell}^{1-2 \alpha} x \\
\Phi_{\ell}^{-\alpha}(0) & =\frac{2 \sin (\pi \alpha)}{\pi} \theta_{\ell}^{1-2 \alpha} \int_{-\infty}^{0} e^{\tau \theta_{\ell}^{2}} x(\tau) \mathrm{d} \tau .
\end{aligned}
$$

The method used to obtain the quadrature nodes $\theta_{\ell}$ and weights $\mu_{\ell}$ is the same as in [7-9]. Following Eq. (22), the Fourier symbol $\chi$ of the operator $D^{\alpha}$ is

$$
\chi(\omega):=(i \omega)^{\alpha} .
$$

On the other hand, for the fractional derivative, the Fourier transform of each differential equation (35a) yields the Fourier symbol $\tilde{\chi}$ of the diffusive approximation:

$$
\tilde{\chi}(\omega):=\frac{2 \sin (\pi \alpha)}{\pi} i \omega \sum_{\ell=1}^{n_{\phi}} \frac{\mu_{\ell} \theta_{\ell}^{2 \alpha-1}}{\theta_{\ell}^{2}+i \omega} .
$$


A nonlinear optimization method (Shor's iterative method) is used to minimize the relative error on Fourier symbols

$$
\left|\frac{\tilde{\chi}(\omega)}{\chi(\omega)}-1\right|
$$

for $\omega$ in an optimization range $\left[\omega_{\min }, \omega_{\max }\right]$, with a positivity constraint on weights $\mu_{\ell}$.

The procedure is very similar for fractional integrals. Following Eq. (C.10), the Fourier symbol of the diffusive approximation is now

$$
\tilde{\chi}(\omega):=\frac{2 \sin (\pi \alpha)}{\pi} \sum_{\ell=1}^{n_{\phi}} \frac{\mu_{\ell} \theta_{\ell}^{1-2 \alpha}}{\theta_{\ell}^{2}+i \omega}
$$

\section{AppendixD. Initial condition for the diffusive approximation}

For $x$ a periodic function of zero mean, the diffusive approximation functions $\Phi_{\ell}^{-\alpha}$ for the fractional integral $D^{-\alpha} x$ are defined by the differential system (C.10), and for $x$ a Fourier sum of zero mean

$$
x(\tau)=\sum_{k=1}^{H} x_{c k} \cos (k \omega \tau)+x_{s k} \sin (k \omega \tau)
$$

the initial condition (C.10b) can be written:

$$
\Phi_{\ell}^{-\alpha}(0)=\frac{2 \sin (\pi \alpha)}{\pi} \theta_{\ell}^{1-2 \alpha} \sum_{k=1}^{H} \frac{\theta_{\ell}^{2} x_{c k}-k \omega x_{s k}}{\theta_{\ell}^{4}+k^{2} \omega^{2}}
$$

Proof. Starting from Eq. (C.10b) and substituting for $x$ the Fourier sum (D.1),

$$
\begin{aligned}
\Phi^{-\alpha}(\theta, 0) & =\frac{2 \sin (\pi \alpha)}{\pi} \theta^{1-2 \alpha} \int_{-\infty}^{0} e^{\tau \theta^{2}} \sum_{k=1}^{H} x_{c k} \cos (k \omega \tau)+x_{s k} \sin (k \omega \tau) \mathrm{d} \tau \\
& =\frac{2 \sin (\pi \alpha)}{\pi} \theta^{1-2 \alpha} \sum_{k=1}^{H} x_{c k}\left(\int_{-\infty}^{0} e^{\tau \theta^{2}} \cos (k \omega \tau) \mathrm{d} \tau\right)+x_{s k}\left(\int_{-\infty}^{0} e^{\tau \theta^{2}} \sin (k \omega \tau) \mathrm{d} \tau\right)
\end{aligned}
$$

Substituting in Eq. (D.4) the real and imaginary parts of:

$$
\int_{-\infty}^{0} e^{\tau \theta^{2}} \cos (k \omega \tau) \mathrm{d} \tau+i \int_{-\infty}^{0} e^{\tau \theta^{2}} \sin (k \omega \tau) \mathrm{d} \tau=\int_{-\infty}^{0} e^{\tau \theta^{2}} e^{i k \omega \tau} \mathrm{d} \tau=\frac{1}{\theta^{2}+i k \omega}=\frac{\theta^{2}-i k \omega}{\theta^{4}+k^{2} \omega^{2}}
$$

yields the new formulation (D.2) of the initial condition (C.10b).

Similarly, for $x$ a Fourier sum

$$
x(\tau)=x_{0}+\sum_{k=1}^{H} x_{c k} \cos (k \omega \tau)+x_{s k} \sin (k \omega \tau)
$$

the diffusive approximation functions $\Phi_{\ell}$ for the fractional derivative $D^{\alpha} x$ are defined by the differential Eq. (35a) with the initial condition (35b), now written:

$$
\Phi_{\ell}(0)=\frac{2 \sin (\pi \alpha)}{\pi} \theta_{\ell}^{2 \alpha-1} \sum_{k=1}^{H} \frac{k \omega\left(\theta_{\ell}^{2} x_{s k}+k \omega x_{c k}\right)}{\theta_{\ell}^{4}+k^{2} \omega^{2}}
$$

The $n_{\phi}$ ordinary differential equations on $\Phi_{\ell}$ (Eq. (35a) for the derivative, Eq. (C.10a) for the integral) are added to the differential system. The functions $\Phi_{\ell}$ are decomposed on $H$ harmonics and the harmonic 
balance is applied, but with the same adjustment as in [31] : the equation derived from balancing the constant ("harmonic 0") of the differential equation must be replaced with the appropriate initial condition (Eq. (D.2) for the integral, Eq. (D.7) for the derivative). Moreover, this equation must comply with the ANM formalism, i.e. it must be recast as a quadratic polynomial system.

We now make this recast explicit for the fractional integral. To this end, we define

$$
\forall \ell \in \llbracket 1, n_{\phi} \rrbracket, \forall k \in \llbracket 1, H \rrbracket, \quad f_{\ell k}:=\frac{\theta_{\ell}^{2} x_{c k}-k \omega x_{s k}}{\theta_{\ell}^{4}+k^{2} \omega^{2}}=\frac{\frac{1}{\theta_{\ell}^{2}} x_{c k}-\frac{k}{\theta_{\ell}^{4}} \omega x_{s k}}{1+\frac{k^{2}}{\theta_{\ell}^{4}} \omega^{2}}
$$

These $n_{\phi} H$ auxiliary unknowns $f_{\ell k}$ are added to the usual Fourier unknowns. The auxiliary unknown

$$
\Omega:=\omega^{2}
$$

renders Eq. (D.8) quadratical:

$$
f_{\ell k}-\frac{1}{\theta_{\ell}^{2}} x_{c k}+\frac{k^{2}}{\theta_{\ell}^{4}} \Omega f_{\ell k}+\frac{k}{\theta_{\ell}^{4}} \omega x_{s k}=0
$$

Then, evaluating the Fourier series of $\Phi_{\ell}^{-\alpha}$ at $t=0$, each equation (D.2) is linear:

$$
\sum_{k=0}^{H} \Phi_{\ell, c k}^{-\alpha}-\frac{2 \sin (\pi \alpha)}{\pi} \theta_{\ell}^{1-2 \alpha} \sum_{k=1}^{H} f_{\ell k}=0
$$

The $n_{\phi}$ functions introduced in the differential system are represented with $n_{\phi}(2 H+1)$ Fourier coefficients; and the initial conditions have been reformulated above with $n_{\phi} H+1$ unknowns.

The same type of quadratic recast can be applied to (D.7), with a few more unknowns and equations because of an extra factor $k \omega$ in the numerators.

\section{References}

[1] K. Shibata, A. Takahashi, T. Shirai: Fault diagnosis of rotating machinery through visualisation of sound signals. Mechanical Systems and Signal Processing, 14 (2), p. 229-241, 2000.

[2] A. Chaigne, J. Kergomard: Acoustics of Musical Instruments (Vol. 844). Springer, 2016.

[3] E. L. Allgower, K. Georg: Introduction to Numerical Continuation Methods. Society for Industrial and Applied Mathematics, 2003.

[4] B. Cochelin, C. Vergez: A high order purely frequency-based harmonic balance formulation for continuation of periodic solutions. Journal of Sound and Vibration, 324, p. 243-262, 2009.

[5] J. H. Smith, J. Woodhouse: The tribology of rosin. Journal of the Mechanics and Physics of Solids, 48 (8), p. 1633-1681, 2000.

[6] P. Vigué. Solutions périodiques et quasi-périodiques de systèmes dynamiques d'ordre entier ou fractionnaire - Applications à la corde frottée. PhD dissertation, Aix-Marseille Université, 2017.

[7] H. Berjamin, B. Lombard, C. Vergez, E. Cottanceau: Time-domain numerical modeling of brass instruments including nonlinear wave propagation, viscothermal losses, and lips vibration. Acta Acustica united with Acustica, 103 (1), p. 117-131, 2017.

[8] B. Lombard, D. Matignon: Diffusive approximation of a time-fractional Burger's equation in nonlinear acoustics. SIAM Journal on Applied Mathematics, 76 (5), p. 1765-1791, 2016.

[9] O. Richoux, B. Lombard, J.F. Mercier: Generation of acoustic solitary waves in a lattice of Helmholtz resonators. Wave Motion 56, p. 85-99, 2015.

[10] R. L. Bagley, P. J. Torvik: A theoretical basis for the application of fractional calculus to viscoelasticity. Journal of Rheology, 27 (3), p. 201-210, 1983. 
[11] R. Metzler, E. Barkai, J. Klafter: Anomalous diffusion and relaxation close to thermal equilibrium: A fractional Fokker-Planck equation approach. Physical Review Letters, 82 (18), p. 3563-3567, 1999.

[12] D. A. Benson, S. W. Wheatcraft, M. M. Meerschaert: Application of a fractional advection-dispersion equation. Water Resources Research, 36 (6), p. 1403-1412, 2000.

[13] Y. A. Rossikhin, M. V. Shitikova: Application of fractional calculus for dynamic problems of solid mechanics: novel trends and recent results. Applied Mechanics Reviews, 63 (1), 010801(1-52), 2010.

[14] R. Hilfer: Applications of Fractional Calculus in Physics. World Scientific, 2000.

[15] C. Y. Zhang: Pseudo almost periodic solutions of some differential equations. Journal of Mathematical Analysis and Applications 181 (1), p. 62-76, 1994.

[16] R. Agarwal, B. de Andrade, C. Cuevas: On type of periodicity and ergodicity to a class of fractional order differential equations. Advances in Difference Equations 1, p. 1-25, 2010.

[17] R. Agarwal, C. Cuevas, H. Soto, M. El-Gebeily: Asymptotic periodicity for some evolution equations in Banach spaces. Nonlinear Analysis: Theory, Methods \& Applications 74(5), p. 1769-1798, 2011.

[18] C. Lizama, G. M. N'Guérékata: Mild solutions for abstract fractional differential equations. Applicable Analysis 92 (8), p. 1731-1754, 2013.

[19] V. Mishra, S. Das, H. Jafari, SH Ong: Study of fractional order van der Pol equation. Journal of King Saud University-Science 28(1), p. 55-60, 2016.

[20] M. Belmekki, J. Nieto, R. Rodriguez-Lopez: Existence of periodic solution for a nonlinear fractional differential equation. Boundary Value Problems 1, p. 1-18, 2009.

[21] K. Diethelm, N. J. Ford, A. D. Freed: A predictor-corrector approach for the numerical solution of fractional differential equations. Nonlinear Dynamics, 29 (1), p. 3-22, 2002.

[22] M. Aoun, R. Malti, F. Levron, A. Oustaloup: Numerical simulations of fractional systems: an overview of existing methods and improvements. Nonlinear Dynamics, 38 (1-4), p. 117-131, 2004.

[23] P. Kumar, O. P. Agrawal: An approximate method for numerical solution of fractional differential equations. Signal Processing, 86 (10), p. 2602-2610, 2006.

[24] A. H. Bhrawy, T. M. Taha, J. A. T. Machado: A review of operational matrices and spectral techniques for fractional calculus. Nonlinear Dynamics 81 (3), p. 1023-1052, 2015.

[25] R. Hilfer: Threefold Introduction to Fractional Derivatives. Anomalous Transport: Foundations and Applications, p. 17-73, 2008.

[26] M. D. Ortigueira: Fractional Calculus for Scientists and Engineers. Vol. 84. Springer, 2011.

[27] I. Podlubny: Fractional differential equations: an introduction to fractional derivatives, fractional differential equations, to methods of their solution and some of their applications (Vol. 198). Academic press, 1998.

[28] I. Petráš: Fractional-order nonlinear systems: modeling, analysis and simulation. Springer Science \& Business Media, 2011.

[29] A. A. Kilbas, H. M. Srivastava, J. J. Trujillo: Theory and Applications of Fractional Differential Equations, Volume 204, Elsevier Science, 2006.

[30] K. Diethelm: An investigation of some nonclassical methods for the numerical approximation of Caputotype fractional derivatives. Numer. Algor. 47, p. 361-390, 2008.

[31] S. Karkar, B. Cochelin, C. Vergez: A high-order, purely frequency based harmonic balance formulation for continuation of periodic solutions: The case of non-polynomial nonlinearities. Journal of Sound and Vibration 332 (4), p. 968-977, 2013. 
[32] L. Guillot, P. Vigué, C. Vergez, B. Cochelin: Continuation of quasi-periodic solutions with two-frequency Harmonic Balance Method, Journal of Sound and Vibration, 394, p. 434-450, 2017.

[33] E. Kaslik, S. Sivasundaram: Non-existence of periodic solutions in fractional-order dynamical systems and a remarkable difference between integer and fractional-order derivatives of periodic functions. Nonlinear Analysis: Real World Applications, 13 (3), p. 1489-1497, 2012.

[34] J. Wang, M. Feckan, Y. Zhou: Nonexistence of periodic solutions and asymptotically periodic solutions for fractional differential equations. Communications in Nonlinear Science and Numerical Simulation, 18 (2), p. 246-256, 2013.

[35] I. Area, J. Losada, J. J. Nieto: On fractional derivatives and primitives of periodic functions. Abstract and Applied Analysis, Hindawi, 2014.

[36] C. M. Pinto, J. A. T. Machado: Complex-order forced Van der Pol oscillator. Journal of Vibration and Control, 18 (14), p. 2201-2209, 2012.

[37] M. S. Tavazoei, M. Haeri, M. Attari, S. Bolouki, M. Siami: More details on analysis of fractional-order van der Pol oscillator. Journal of Vibration and Control, 15 (6), p. 803-819, 2009.

[38] J. H. Chen, W. C. Chen: Chaotic dynamics of the fractionally damped van der Pol equation. Chaos, Solitons \& Fractals, 35 (1), p. 188-198, 2008.

[39] T. Sardar, S. S. Ray, R. K. Bera, B. B. Biswas: The analytical approximate solution of the multi-term fractionally damped van der Pol equation. Physica Scripta, 80 (2), p. 025003 (6 pages).

[40] A. Lazarus, O. Thomas: A harmonic-based method for computing the stability of periodic solutions of dynamical systems. Comptes Rendus Mecanique, 338 (9), p. 510-517, 2010.

[41] D. Matignon: Stability results for fractional differential equations with applications to control processing. Computational engineering in systems applications, 2, p. 963-968, 1996.

[42] E. Kaslik, S. Sivasundaram: Nonlinear dynamics and chaos in fractional-order neural networks. Neural Networks, 32, p. 245-256, 2012.

[43] E. Cuesta: Asymptotic behaviour of the solutions of fractional integro-differential equations and some time discretizations. Discrete and Continuous Dynamical Systems, Supplement 2007, p. 277-285. 